\title{
ON NONMATERIAL SURFACES WITH STRUCTURE
}

\author{
BY \\ NANCY M. PFENNING AND W. O. WILLIAMS \\ Carnegie Mellon University, Pittsburgh, Pennsylvania
}

1. Introduction. In the development of continuum theories of mechanics and thermodynamics, nonstandard formulations often are difficult to motivate and/or to justify. Even if it is possible to add the desired terms to more conventional theories without violating physical laws, this usually raises questions regarding the implicit exclusion of other, perhaps equally appropriate, terms or regarding the particular choices of format of the terms. A straightforward way of dealing with some of these questions is to rephrase the assumptions of the theory in global form, putting the fundamental assumptions in terms that one hopes are closer to experience or experiment. This line of development can be traced back to the beginning of continuum theory but is traditionally associated with the name of Cauchy. Noll [N1] inaugurated the modern approach, and he was followed in his study of conventional continuum theory by Gurtin and Williams [GW] and Fischer and Leitman [FL] and more recently by Gurtin and Martins [GMa], Gurtin, Williams, and Ziemer [GWZ], Śilhavý [S1], and others. In the more controversial models, which include nontraditional kinematics, there have been applications to various sorts of mixture theories and structured continua. One area that involves only moderate structure and that seems very appropriate to applications of this method is that of a continuum which carries a moving surface having a distinct structure. Leitman and Fischer [LF] inaugurated such a study in the case of surfaces that are material, that is, that move with the motion of the continuum, and this was pursued by Gurtin and Murdoch [GMu]. The formal treatment of nonmaterial surfaces was considered in part by Williams [W] and continued by Pfenning [P]. This paper is an extension of the last two works. In both [W] and [P] only classical balance equations were considered, but this led to a set of equations that clearly were inadequate to model such situations as phase transitions; in [P] it was found that the notion of surface tension, central to many physical models of phase boundaries, was not compatible with the notion that the transition surface was nonmaterial. In this work we go further, considering, as in $[\mathrm{P}]$, the three-dimensional body and the surface to be distinct objects and deriving separate balance and interaction equations, but here allowing a more rich interaction between the two than appears in [P], and we are led to a second set of balance relations and the reintroduction of the equivalent of a surface tension. The idea of

Received October 21, 1991.

1991 Mathematics Subject Classification. Primary 53A05, 73B30, 80A20.

(C)1993 Brown University 
separate balances was suggested to us by the recent work of Gurtin and Struthers [GS,G], who use a quasi-formal presentation of the theory. In that work the second set of balance equations is introduced via a notion of frame-indifference of the reference motion; we prefer to regard the corresponding equations as arising from a principle of symmetry and believe that our derivation is more straightforward. In fact, the physical quantities that we find (the material stress tensor, etc.) do not correspond exactly to those of [GS], although there are obvious correspondences and parallels, and the resulting balance equations are rather similar. ${ }^{1}$

We consider the situation in which a surface of transition traverses a body, with possibly profound changes in the physical structure of the body occurring across the surface, modeling the solidification of a crystalline solid from a melt or flow through a shock wave. Formally, we choose to regard the body as a structured continuum: a partial superposition of the two bulk bodies and of the two-dimensional surface. The interactions among the three are modeled in standard ways, but we allow the power function to depend upon all the various velocities. The classical force and moment balance equations are derived based on a principle of frame-indifference [N2, GR], and the nonclassical equations from a principle of invariance. ${ }^{2}$

While the significance of a formal theory such as this is that the axioms are complete and the results derivable with no further assumptions, the mathematics involved for the proofs and derivations is not difficult and follows from that in [W] and [Pf] so that we feel justified in including at most sketches of proofs, emphasizing instead the unique features of the representations.

2. Kinematics, structure. Our setting is 3-dimensional Euclidean space $\mathscr{E}$ with translation space $\mathscr{V}$. The material body $\mathscr{B}$ is a smooth region (regularly closed, with piecewise smooth boundary), which is divided into two smooth regions $\mathscr{B}_{+}$ and $\mathscr{B}_{-}$by a surface $\mathscr{S}$. N the normal vector to $\mathscr{S}$, is chosen to point from $\mathscr{B}_{-}$into $\mathscr{B}_{+}$. We consider collections of smooth closed subsets of $\mathscr{B}_{+}$and of $\mathscr{B}_{-}$ and smooth closed subsets of $\mathscr{S}$. We make the distinction that this is a structured continuum by considering $\mathscr{B}_{+}, \mathscr{B}_{-}$, and $\mathscr{S}$ as distinct bodies, which happen to coincide in position. In particular, if a part $\mathscr{P}$ includes a part $\mathscr{R}$ of $\mathscr{S}$ on its boundary, we must distinguish between $\mathscr{R}$ as a part of $\partial \mathscr{P}$ and $\mathscr{R}$ as an element of the material universe which may interact with $\mathscr{P}$. To simplify notation, we will suppose that $\mathscr{S}$ shares no area with $\partial \mathscr{B}$.

This is emphasized by the fundamental assumption which we make, that the material collection $\mathscr{M}$ has associated to it a vector space $\mathbb{F}$ of velocity fields $\mathbf{V}:=$ $\left(\mathbf{v}_{+}, \mathbf{v}_{-}, \mathbf{v}\right)$, where $\mathbf{v}_{ \pm}$is defined on $\mathscr{B}_{ \pm}$, respectively, and $\mathbf{v}$ is defined on $\mathscr{S}$. This is the field of velocities on which the power functions act. Note that $\mathbf{v}_{ \pm}$is

\footnotetext{
${ }^{1}$ Our presentation differs from that of [GS] in that we do not introduce reference configurations and material description of the motion; we work only in the current configuration, which allows great simplification of notation. To formulate constitutive equations for solid bodies it is necessary to introduce such concepts and to connect them to the "Eulerian" kinematics, which we utilize. Cf. Sec. 9.

${ }^{2}$ This corresponds to the principle of invariance under change of lattice observers of Gurtin and Struthers; there is no practical difference in this case, but in general our point of view, based on a notion of material symmetry, may prove more restrictive in other situations.
} 
supposed to be defined on $\mathscr{S}$ as well as in the interior of $\mathscr{B}_{ \pm}$. In the next section we shall introduce the assumption that the power exerted on an element acts upon the velocity defined on that element and, hence, that the power functions associated to each of $\mathscr{P}$ and $\mathscr{R}$ may involve all of the three fields $\mathbf{v},\left.\mathbf{v}_{+}\right|_{\mathscr{S}}$, and $\left.\mathbf{v}_{-}\right|_{\mathscr{S}}$. Formally, we require that each field be $C^{1}$ on its domain, including the boundary. ${ }^{3}$ Of course it is essential to allow the bulk velocities to differ on the surface $\mathscr{S}$, subject to the law of balance of mass, in view of the applications we have in mind. In these cases, there is a transfer of substance between the bodies, and thus velocities at the surface may differ in any manner. It is worth noting that, since the normal vector $\mathbf{N}$ points from $\mathscr{B}_{-}$to $\mathscr{B}_{+}$, positive values of $\left(\mathbf{v}-\mathbf{v}_{+}\right) \cdot \mathbf{N}$ correspond to loss of volume of $\mathscr{B}_{+}$, and negative values to gain of volume; the opposite rule governs for $\mathscr{B}_{-}$. One constitutive constraint that will enter into our considerations is that the surface will be supposed to be nonmaterial, that particles on $\mathscr{S}$ have no physical identity, which will require the effective part of $\mathbf{v}$ to be parallel to $\mathbf{N}$. This requirement means that the motion of the surface parallel to itself is only a mathematical construct: there is no reason to identify apparent motion of the surface parallel to itself as having physical significance, as there is no associated movement of particles and hence no change of state of the system. Normal motion of the surface, on the other hand, has a natural physical interpretation. ${ }^{4}$

We require, moreover, that the space of velocities includes all rigid velocity fields: fields of the form $\left(R(\mathrm{c}, \Omega)^{3}\right)$ where

$$
R(x)=\mathbf{c}+\Omega(x-z)
$$

with $\mathrm{c}$ any given vector, $\Omega$ any given skew tensor, and $z$ any point of $\mathscr{E}$. Likewise we will require, for any $\left(\mathbf{v}, \mathbf{v}_{+}, \mathbf{v}_{-}\right) \in \mathbb{F}$ and any rigid field $R(\mathbf{c}, \Omega)$, that $\left(\mathbf{v}+R(\mathbf{c}, \Omega), \mathbf{v}_{+}, \mathbf{v}_{-}\right) \in \mathbb{F}$.

3. Mass, energy, entropy, heat, and power. In this section we shall find representations for these quantities (denoted $M, E, S, H$, and $P$, respectively) based on bounds on additive set functions on the material universe $\mathscr{M}$. These follow immediately from results in [W] and [GW], and so we will only discuss the aspects of the results that are peculiar to the current formulation. In particular, we will present no proofs; see [P] for verifications.

\subsection{Assumption. We suppose given}

$$
\begin{aligned}
M & : \mathscr{M} \rightarrow \mathbb{R}, \\
E & : \mathscr{M} \rightarrow \mathbb{R}, \\
S & : \mathscr{M} \rightarrow \mathbb{R}, \\
H & :\left(\mathscr{M} \times \mathscr{M}^{\mathrm{e}}\right)_{\mathrm{dis}} \rightarrow \mathbb{R}, \quad P:\left(\mathscr{M} \times \mathscr{M}^{\mathrm{e}}\right)_{\mathrm{dis}} \times \mathbb{F} \rightarrow \mathbb{R},
\end{aligned}
$$

where $M, E$, and $S$ are additive, $H$ is biadditive, and $P$ is biadditive in its first

\footnotetext{
${ }^{3}$ The choice is made for convenience; as with the smoothness assumed for the sets involved, one can broaden all concepts to weaker formulations: the basic results will not change.

${ }^{4}$ The first systematic use of this observation apparently was by Moeckel [M].
} 
two arguments, linear in its last argument. Here the notation $(\cdot)_{\text {dis }}$ indicates disjoint pairs from the product.

These are standard assumptions for the mass, energy, entropy, heat flux, and power functions. The representation of these set functions in terms of integrals follows directly from bounds for them in terms of volume $V$, area $A$, and length $L{ }^{5}$.

To save over-elaborate notation and quantifications, let us agree that $\mathscr{P}$ and $\mathscr{Q}$ are to be parts of $\mathscr{B}_{ \pm}$, and $\mathscr{R}$ and $\mathscr{T}$ parts of $\mathscr{S}$. The statements below are presumed to be valid for each choice of $\mathscr{P}, \mathscr{Q}$, and $\mathscr{R}$, unless other restrictions are stated. The additivity assumptions made above allow us to specify bounds in terms of these quantities alone, leaving aside compound elements which are unions of sets from $\mathscr{B}_{+}, \mathscr{B}_{-}$, or $\mathscr{S}$.

3.2. Assumption. There exist scalars $\alpha, \beta, \gamma, \delta$ such that for all admissible values of the arguments

$$
\begin{aligned}
|M(\mathscr{P})| & \leq \alpha V(\mathscr{P}), \\
|E(\mathscr{P})| & \leq \alpha V(\mathscr{P}), \\
|S(\mathscr{P})| & \leq \alpha V(\mathscr{P}), \\
|H(\mathscr{P}, \mathscr{Q})| & \leq \gamma A(\mathscr{P} \cap \mathscr{Q} \mid \mathscr{S}), \\
|H(\mathscr{P}, \mathscr{B})| & \leq \gamma A(\mathscr{P} \cap \partial \mathscr{B})+\delta V(\mathscr{P}), \\
|P(\mathscr{P}, \mathscr{Q} ; V)| & \leq \gamma A(\mathscr{P} \cap \mathscr{Q} \backslash \mathscr{S}), \\
\mid P(\mathscr{P}, \mathscr{B} & \left.\mathscr{e}^{\mathrm{e}} V\right) \mid \leq \gamma A(\mathscr{P} \cap \partial \mathscr{B})+\delta V(\mathscr{P}), \\
|E(\mathscr{R})| & \leq \beta A(\mathscr{R}), \\
|H(\mathscr{R}, \mathscr{T})| & \leq \gamma L(\mathscr{R} \cap \mathscr{T}), \\
\mid H(\mathscr{R}, \mathscr{B} & \mathbf{e}) \mid \leq \gamma L(\mathscr{R} \cap \partial \mathscr{B})+\delta A(\mathscr{R}), \\
|P(\mathscr{R}, \mathscr{T} ; V)| & \leq \gamma L(\mathscr{R} \cap \mathscr{T}), \\
\mid P(\mathscr{R}, \mathscr{B} & \left.{ }^{\mathrm{e}} ; V\right) \mid \leq \gamma L(\mathscr{R} \cap \partial \mathscr{B})+\delta V(\mathscr{R}),
\end{aligned}
$$

and for the surface-solid interactions

$$
\begin{aligned}
|H(\mathscr{R}, \mathscr{P})| & \leq \gamma A(\mathscr{R} \cap \mathscr{P}), \\
|H(\mathscr{P}, \mathscr{R})| & \leq \gamma A(\mathscr{P} \cap \mathscr{R}), \\
|P(\mathscr{R}, \mathscr{P} ; V)| & \leq \gamma A(\mathscr{R} \cap P), \\
|P(\mathscr{P}, \mathscr{R} ; V)| & \leq \gamma A(\mathscr{P} \cap \mathscr{R}) .
\end{aligned}
$$

Remark. Note that we require that regions on opposite sides of the surface $\mathscr{S}$ exchange heat and power only by using $\mathscr{S}$ as a courier; under our structure such elements are not considered to be in contact. Leaving aside the terms that enforce this requirement, the bounds are in familiar form.

These bounds lead by standard arguments to integral representations for each

\footnotetext{
${ }^{5}$ As proved by Šilhavý in [S2], it is sufficient to assume that the bounds be expressible in terms of integrals of integrable functions.
} 
quantity, as we illustrate by first giving those for $M, E, S$, and $H$ :

$$
\begin{aligned}
M(\mathscr{P}) & =\int_{\mathscr{P}} \rho d V, & M(\mathscr{R}) & =0, \\
E(\mathscr{P}) & =\int_{\mathscr{P}} E d V, & E(\mathscr{R}) & =\int_{\mathscr{R}} \varepsilon d A, \\
S(\mathscr{P}) & =\int_{\mathscr{P}} S d V, & S(\mathscr{R}) & =\int_{\mathscr{R}} \eta d A
\end{aligned}
$$

and

$$
\begin{aligned}
H(\mathscr{P}, \mathscr{Q}) & =\int_{\partial \mathscr{P} \cap \mathscr{Q}} q_{\partial \mathscr{P}} d A, & H\left(\mathscr{P}, \mathscr{B}^{\mathrm{e}}\right) & =\int_{\partial \mathscr{P} \cap \mathscr{B}^{\mathrm{e}}} q_{\partial \mathscr{B}} d A+\int_{\mathscr{P}} r d V, \\
H(\mathscr{P}, \mathscr{R}) & =\int_{\mathscr{D} \cap \mathscr{R}} q_{\mathscr{B}}{ }^{ \pm} d A, & H(\mathscr{R}, \mathscr{P}) & =\int_{\mathscr{R} \cap \mathscr{P}} q_{\mathscr{S}^{ \pm}} d A, \\
H(\mathscr{R}, \mathscr{T}) & =\int_{\mathscr{R} \cap \mathscr{T}} q_{\partial \mathscr{R}} d L, & H\left(R, B^{\mathrm{e}}\right) & =\int_{\mathscr{R} \cap \mathscr{B}} q_{\partial \mathscr{S}} d L+\int_{\mathscr{R}} r_{\mathscr{S}} d A .
\end{aligned}
$$

Discussion of the power functions is complicated by the dependence on the velocity field $V$. To simplify discussion we shall assume that the material is nonpolar, anticipating that as a consequence the densities of the power function at a point $x$ will be linear functions of the value of the velocity fields defined at $x$ rather than more general linear functions of the germ of the velocities at $x$ (cf. [W] and [P] for details). ${ }^{6}$ Note also that we choose to replace each linear function $F$ of the absolute velocities by a linear function $G$, which is expressed in terms of the relative velocities:

$$
F\left(\mathbf{v}, \mathbf{v}_{+}, \mathbf{v}_{-}\right)=G\left(\mathbf{v}, \mathbf{v}-\mathbf{v}_{+}, \mathbf{v}-\mathbf{v}_{-}\right) .
$$

Then we have for the power function

$$
\begin{aligned}
& P(\mathscr{P}, \mathscr{Q}, V)=\int_{\partial \mathscr{P} \cap \mathscr{Q}} \mathbf{t}_{\partial \mathscr{P}} \cdot \mathbf{v}_{ \pm} d A, \\
& P(\mathscr{P}, \mathscr{R}, V)=\int_{\mathscr{P} \cap \mathscr{R}} \mathbf{t}_{\mathscr{B}^{ \pm}} \cdot \mathbf{v}_{ \pm}+\boldsymbol{\tau}_{\mathscr{B}^{ \pm}} \cdot\left(\mathbf{v}-\mathbf{v}_{ \pm}\right) d A, \\
& P(\mathscr{R}, \mathscr{P}, V)=\int_{\mathscr{R} \cap \mathscr{P}^{\prime}} \mathbf{t}_{\mathscr{S}^{ \pm}} \cdot \mathbf{v}+\boldsymbol{\tau}_{\mathscr{S}^{ \pm}} \cdot\left(\mathbf{v}-\mathbf{v}_{ \pm}\right) d A, \\
& P(\mathscr{R}, \mathscr{T}, V)=\int_{\mathscr{R} \cap \mathscr{G}} \mathbf{t}_{\partial \mathscr{R}} \cdot \mathbf{v}+\boldsymbol{\tau}_{\partial \mathscr{R}}^{+} \cdot\left(\mathbf{v}-\mathbf{v}_{+}\right)+\boldsymbol{\tau}_{\partial \mathscr{R}}^{-} \cdot\left(\mathbf{v}-\mathbf{v}_{-}\right) d L, \\
& P\left(\mathscr{P}, \mathscr{B}^{\mathrm{e}}, V\right)=\int_{\partial \mathscr{P} \cap \mathscr{B}^{\mathrm{e}}} \mathbf{t}_{\partial \mathscr{B}} \cdot \mathbf{v}_{ \pm} d A+\int_{\mathscr{P}} \mathbf{b} \cdot \mathbf{v}_{ \pm} d V \\
& +\int_{\mathscr{P}_{\cap} \mathscr{S}} \mathbf{b}_{\mathscr{B}^{ \pm}} \cdot \mathbf{v}_{ \pm}+\boldsymbol{\beta}_{\mathscr{B}^{ \pm}} \cdot\left(\mathbf{v}-\mathbf{v}_{ \pm}\right) d A, \\
& P\left(\mathscr{R}, \mathscr{B}^{\mathrm{e}}, V\right)=\int_{\partial \mathscr{R} \cap \mathscr{B}} \mathbf{t}_{\partial \mathscr{S}} \cdot \mathbf{v} d L+\int_{\mathscr{R}} \mathbf{b}_{\mathscr{S}} \cdot \mathbf{v} \\
& +\boldsymbol{\beta}_{\mathscr{S}^{+}} \cdot\left(\mathbf{v}-\mathbf{v}_{+}\right)+\boldsymbol{\beta}_{\mathscr{S}^{-}} \cdot\left(\mathbf{v}-\mathbf{v}_{-}\right) d A \text {. }
\end{aligned}
$$

\footnotetext{
${ }^{6}$ It may occur that couple-stresses are significant in some applications. It is not difficult to add these effects to the theory we present, although the expression of the energy equation becomes much more complex. In [P] Pfenning develops such a theory.
} 
The choice of $\mathbf{v}_{ \pm}$in these equations, of course, is to be in accord with the location $\mathscr{P} \subset \mathscr{B}_{ \pm}$.

Some terms in these representations are relatively standard: the stress vector $\mathbf{t}_{\partial \mathscr{P}}$ represents forces exerted by one part of the bulk body on another, and $\mathbf{t}_{\partial \mathscr{R}}$ is the corresponding surface stress vector, representing forces exerted between parts of the surface. Likewise, b can be the standard body force (e.g., gravity) on the bulk matter, and $\mathbf{b}_{\mathscr{S}}$ the corresponding term on the surface. The other force terms are nonstandard, and we will have to examine them in the context of the balance equations and of the entropy inequality.

As remarked earlier, since we suppose the surface to be nonmaterial, the normal part of $\mathbf{v}$ is the only part that has physical significance. Thus we shall require that the working be the same for velocity triples $\left(\mathbf{v}, \mathbf{v}_{+}, \mathbf{v}_{-}\right)$and $\left(\overline{\mathbf{v}}, \overline{\mathbf{v}}_{+}, \overline{\mathbf{v}}_{-}\right)$for which

$$
\mathbf{v} \cdot \mathbf{N}=\overline{\mathbf{v}} \cdot \mathbf{N}
$$

and note that, as a consequence, the corresponding force terms $\mathbf{t}_{\mathscr{P}^{ \pm}}, \mathbf{t}_{\partial \mathscr{R}}$, and $\mathbf{b}_{\mathscr{S}}$ are parallel to $\mathbf{N}$.

4. Time rates of change; balance of mass. We now assume that the above set functions are specified as part of a (smooth) process parameterized by time $t$ and examine the form that the corresponding derivatives take. All densities thus are functions of position and of time, and the surface evolves in time as previously noted. We will assume that the densities $\rho, E$, and $S$ are smooth on $\mathscr{B}_{ \pm}$and that $\varepsilon$ and $\eta$ are smooth. Then we consider typical terms

$$
Y(\mathscr{P})=\int_{\mathscr{P}} \Psi d V, \quad Y(\mathscr{R})=\int_{\mathscr{R}} \psi d A
$$

and take the derivative of each term with respect to time. Naturally, we suppose that $\mathscr{P}$ is transported by the velocity field $\mathbf{v}_{+}$or $\mathbf{v}_{-}$as appropriate, and since the surface $\mathscr{S}$ does not have a material (standing) identity, we will compute the derivative as following the normal velocity field $\mathbf{v} \cdot \mathbf{N}$. (To use another velocity would introduce spurious transfer terms, which then would be balanced with corresponding terms introduced for the power and heat functions and so finally eliminated.)

With this in mind we calculate that

$$
\dot{Y}(\mathscr{P}):=\frac{d}{d t} Y(\mathscr{P})=\int_{\mathscr{P}} \dot{\Psi}+\Psi \operatorname{div} \mathbf{v}_{ \pm} d V \mp \int_{\mathscr{P} \cap \mathscr{P}} \Psi_{ \pm}\left(\mathbf{v}-\mathbf{v}_{ \pm}\right) \cdot \mathbf{N} d A
$$

and

$$
\dot{Y}(\mathscr{R}):=\frac{d}{d t} Y(\mathscr{R})=\int_{\mathscr{R}} \stackrel{\circ}{\psi}-\psi \kappa \mathbf{v} \cdot \mathbf{N} d A .
$$

Here the superposed dot in $\dot{\Psi}$ denotes the usual material or transported derivative, while the circle in $\stackrel{\circ}{\psi}$ denotes the corresponding derivative of $\psi$ following the normal transport of the surface. $\Psi_{ \pm}$denotes the trace of the function on the appropriate side of the surface $\mathscr{S}$. We use $\kappa$ to denote twice the mean curvature (cf. [GM]) and recall that our convention has $\mathbf{N}$ pointing from $\mathscr{B}_{-}$to $\mathscr{B}_{+}$.

We apply this first to the law of balance of mass:

$$
\frac{d}{d t} M(\mathscr{P})=0 \text {. }
$$


Since this holds for each element of the collection, we see that for $V$-almost every point in $\mathscr{B} \backslash \mathscr{S}$,

$$
\dot{\rho}+\rho \operatorname{div} \mathbf{v}_{ \pm}=0
$$

while $A$-almost everywhere on the surface, since the surface has no mass, balance of mass flux takes the form

$$
\rho_{+}\left(\mathbf{v}-\mathbf{v}_{+}\right) \cdot \mathbf{N}=\rho_{-}\left(\mathbf{v}-\mathbf{v}_{-}\right) \cdot \mathbf{N} .
$$

Notice that only the normal component of the relative velocities enters. We shall call the term

$$
M:=\rho_{+}\left(\mathbf{v}-\mathbf{v}_{+}\right) \cdot \mathbf{N}=\rho_{-}\left(\mathbf{v}-\mathbf{v}_{-}\right) \cdot \mathbf{N}
$$

the mass transport across the surface.

To obtain compact representations for the densities of the derivatives, we shall choose the traditional format for the energy and entropy functions. Assuming that $\rho$ is strictly positive, we may choose to write $E$ and $S$ in the alternative form

$$
\begin{aligned}
& E(\mathscr{P})=\int_{\mathscr{P}} \rho e d V, \\
& S(\mathscr{P})=\int_{\mathscr{P}} \rho s d V,
\end{aligned}
$$

for the parts of $\mathscr{B} \backslash \mathscr{S}$, leaving the form for the parts of the surface as written. We then obtain the derivatives in the form

$$
\begin{aligned}
& \dot{E}(\mathscr{P})=\int_{\mathscr{P}} \rho \dot{e} d V \mp \int_{\mathscr{P} \cap \mathscr{P}} M e_{ \pm} d A, \\
& \dot{S}(\mathscr{P})=\int_{\mathscr{P}} \rho \dot{s} d V \mp \int_{\mathscr{P} \cap \mathscr{S}} M s_{ \pm} d A, \\
& \dot{E}(\mathscr{R})=\int_{\mathscr{R}} \stackrel{\circ}{\varepsilon}-\varepsilon \kappa \mathbf{v} \cdot \mathbf{N} d A, \\
& \dot{S}(\mathscr{R})=\int_{\mathscr{R}} \stackrel{\circ}{\eta}-\eta \kappa \mathbf{v} \cdot \mathbf{N} d A
\end{aligned}
$$

5. Forces due to momentum transfer. The formal structure that we have introduced leaves the momentum terms of the force balance as part of the working effect of the external world $\mathscr{B}^{\mathrm{e}}$ (D'Alembert's principle). To analyze the other terms it is useful to reintroduce the traditional notation for these. Since we do not introduce any esoteric forms of mass, the momentum terms are traditional: the rate of change of the kinetic energy for a general subbody $\mathscr{A}$ is

$$
\begin{aligned}
\frac{d}{d t}\left[\int_{\mathscr{A} \cap \mathscr{B}+} \frac{1}{2} \rho_{+}\left(\mathbf{v}_{+}\right)^{2}+\int_{\mathscr{A} \cap \mathscr{B}-} \frac{1}{2} \rho_{-}\left(\mathbf{v}_{-}\right)^{2}\right] \\
=\int_{\mathscr{A} \cap \mathscr{B}+} \rho_{+}\left(\dot{\mathbf{v}}_{+}\right) \cdot \mathbf{v}_{+}+\int_{\mathscr{A} \cap \mathscr{B}-} \rho_{-}\left(\dot{\mathbf{v}}_{-}\right) \cdot \mathbf{v}_{-} \\
\quad+\int_{\mathscr{A} \cap \mathscr{S}} \frac{1}{2} \rho_{-}\left(\mathbf{v}_{-}\right)^{2}\left(\mathbf{v}-\mathbf{v}_{-}\right) \cdot \mathbf{N}-\frac{1}{2} \rho_{+}\left(\mathbf{v}_{+}\right)^{2}\left(\mathbf{v}-\mathbf{v}_{+}\right) \cdot \mathbf{N} .
\end{aligned}
$$

Examining the cases $\mathscr{A} \subset \mathscr{B}_{+}$and $\mathscr{A} \subset \mathscr{B}_{-}$and comparing to the representation for $P\left(\mathscr{A}, \mathscr{B}^{\mathrm{e}}, V\right)$ suggests that

$$
\mathbf{b}=\mathbf{b}^{\mathrm{e}}-\rho_{ \pm} \dot{\mathbf{v}}_{ \pm} .
$$


Here $\mathbf{b}^{\mathrm{e}}$ is to be the external body force. For the surface term in $P\left(\mathscr{A}, \mathscr{B}^{\mathrm{e}}, V\right)$ we assume $^{7}$

$$
\begin{aligned}
& \mathbf{b}_{\mathscr{B}-}=-\frac{1}{2} \rho_{-}\left(\mathbf{v}-\mathbf{v}_{-}\right) \mathbf{v}_{-}=-\frac{1}{2} M \mathbf{v}_{-}, \\
& \mathbf{b}_{\mathscr{B}+}=\frac{1}{2} \rho_{+}\left(\mathbf{v}-\mathbf{v}_{+}\right) \mathbf{v}_{+}=\frac{1}{2} M \mathbf{v}_{+} .
\end{aligned}
$$

The internal forces generated by the exterior, denoted by $\boldsymbol{\beta}_{\mathscr{F}^{ \pm}}$and $\boldsymbol{\beta}_{\mathscr{S}^{ \pm}}$, may have an effect in the case of massy surfaces, but since it seems unlikely that they enter in the current case we shall assume them to be zero:

$$
\boldsymbol{\beta}_{\mathscr{B}^{ \pm}}=\mathbf{0}, \quad \boldsymbol{\beta}_{\mathscr{S}^{ \pm}}=\mathbf{0} .
$$

6. Balance of forces. In this and the next section we come to the crux of our discussion. To derive the balance of forces one need only invoke a condition of invariance under change of frame for the working (alt. energy balance) over complete systems, as first shown by Noll [N2] and Green and Rivlin [GR]. Thus, for example, we shall require that the function

$$
V \mapsto P\left(\mathscr{P}, \mathscr{P}^{\mathrm{e}}, V\right):=P(\mathscr{P}, \mathscr{B} \backslash \mathscr{P}, V)+P(\mathscr{P}, \mathscr{S}, V)+P\left(\mathscr{P}, \mathscr{B}^{\mathrm{e}}, V\right)
$$

be invariant under superposed rigid motions (changes of frame). We also must treat elements in $\mathscr{S}$.

$$
V \mapsto P\left(\mathscr{R}, \mathscr{R}^{\mathrm{e}}, V\right):=P(\mathscr{R}, \mathscr{S} \backslash \mathscr{R}, V)+P(\mathscr{R}, \mathscr{B}, V)+P\left(\mathscr{P}, \mathscr{B}^{\mathrm{e}}, V\right)
$$

and, in order that compound elements $\mathscr{P} \cup \mathscr{R}$ are also subject to a similar condition we need to require invariance of

$$
V \mapsto P(\mathscr{P}, \mathscr{R}, V)+P(\mathscr{R}, \mathscr{P}, V) .
$$

The resulting calculations deliver the equations of balance of force and of moment for our system.

The significant terms with which we deal are

$$
\begin{aligned}
P\left(\mathscr{P}, \mathscr{P}^{\mathrm{e}}, V\right)= & \int_{\partial \mathscr{P} \backslash \mathscr{S}} \mathbf{t}_{\partial \mathscr{P}} \cdot \mathbf{v}_{ \pm} d A+\int_{\mathscr{P}} \mathbf{b} \cdot \mathbf{v}_{ \pm} d V \\
& +\int_{\mathscr{P} \cap \mathscr{S}^{\prime}} \mathbf{t}_{\mathscr{B}^{ \pm}} \cdot \mathbf{v}_{ \pm}+\boldsymbol{\tau}_{\mathscr{B}^{ \pm}} \cdot\left(\mathbf{v}-\mathbf{v}_{ \pm}\right)+\mathbf{b}_{\mathscr{B}^{ \pm}} \cdot \mathbf{v}_{ \pm}+\boldsymbol{\beta}_{\mathscr{B}^{ \pm}} \cdot\left(\mathbf{v}-\mathbf{v}_{ \pm}\right) d A, \\
P(\mathscr{P}, \mathscr{R}, V)+ & P(\mathscr{R}, \mathscr{P}, V)=\int_{\mathscr{P} \cap \mathscr{R}} \mathbf{t}_{\mathscr{B}}{ }^{ \pm} \cdot \mathbf{v}_{ \pm}+\boldsymbol{\tau}_{\mathscr{B}^{ \pm}} \cdot\left(\mathbf{v}-\mathbf{v}_{ \pm}\right) d A \\
& +\int_{\mathscr{R} \cap \mathscr{P}} \mathbf{t}_{\mathscr{S}^{ \pm}} \cdot \mathbf{v}+\boldsymbol{\tau}_{\mathscr{S}^{ \pm}} \cdot\left(\mathbf{v}-\mathbf{v}_{ \pm}\right) d A,
\end{aligned}
$$

and

$$
\begin{aligned}
P\left(\mathscr{R}, \mathscr{R}^{\mathrm{e}}, V\right)= & \int_{\partial \mathscr{R}} \mathbf{t}_{\partial \mathscr{R}} \cdot \mathbf{v}+\boldsymbol{\tau}_{\partial \mathscr{R}}^{+} \cdot\left(\mathbf{v}-\mathbf{v}_{+}\right)+\boldsymbol{\tau}_{\partial \mathscr{R}}^{-} \cdot\left(\mathbf{v}-\mathbf{v}_{-}\right) d L \\
& +\int_{\mathscr{R}} \mathbf{b}_{\mathscr{S}} \cdot \mathbf{v}+\boldsymbol{\beta}_{\mathscr{S}_{+}} \cdot\left(\mathbf{v}-\mathbf{v}_{+}\right)+\boldsymbol{\beta}_{\mathscr{S}_{-}} \cdot\left(\mathbf{v}-\mathbf{v}_{-}\right) \\
& +\mathbf{t}_{\mathscr{S}_{+}} \cdot \mathbf{v}+\boldsymbol{\tau}_{\mathscr{S}_{+}} \cdot\left(\mathbf{v}-\mathbf{v}_{+}\right)+\mathbf{t}_{\mathscr{S}_{-}} \cdot \mathbf{v}+\boldsymbol{\tau}_{\mathscr{S}_{-}} \cdot\left(\mathbf{v}-\mathbf{v}_{-}\right) d A
\end{aligned}
$$

\footnotetext{
${ }^{7}$ Formally there is another option: to presume that this term acts via $\mathbf{v}-\mathbf{v}_{ \pm}$in the form of the interaction force $\boldsymbol{\beta}_{\mathscr{B}^{ \pm}}$; but this would imply that the momentum transfer vector must be normal to the surface, which in general is not reasonable.
} 
Considering the invariance of total power for $\mathscr{P} \subset \mathscr{B}_{+}$one finds

$$
\int_{\partial \mathscr{P} \backslash \mathscr{S}} \mathbf{t}_{\partial \mathscr{P}} d A+\int_{\partial \mathscr{P} \cap \mathscr{S}} \mathbf{t}_{\mathscr{B}+} d A+\int_{\partial \mathscr{P} \cap \mathscr{S}} \mathbf{b}_{\mathscr{B}+} d A+\int_{\mathscr{P}} \mathbf{b} d V=\mathbf{0}
$$

and

$$
\int_{\partial \mathscr{P} \backslash \mathscr{S}} \mathbf{x} \wedge \mathbf{t}_{\partial \mathscr{P}} d A+\int_{\partial \mathscr{P} \cap \mathscr{S}} \mathbf{x} \wedge \mathbf{t}_{\mathscr{B}+} d A+\int_{\partial \mathscr{P} \cap \mathscr{S}} \mathbf{x} \wedge \mathbf{b}_{\mathscr{B}+} d A+\int_{\mathscr{P}} \mathbf{x} \wedge \mathbf{b} d V=\mathbf{0}
$$

For parts $\mathscr{P}$, which intersect $\mathscr{S}$ at most in zero area, assuming the needed smoothness, this leads to the usual results (cf. [N1, GW]) via hatbox and tetrahedron arguments: there exists a symmetric stress tensor $\mathbf{T}$ on $\mathscr{B}^{ \pm}$that satisfies

$$
\operatorname{div} \mathbf{T}+\mathbf{b}=\mathbf{0}, \quad \mathbf{T n}=\mathbf{t}_{\partial \mathscr{P}},
$$

whenever $\mathbf{n}$ is the exterior normal to $\partial \mathscr{P}$. One may also show (cf. [W]) that the stress tensor may be defined $A$-ae on $\mathscr{S}$ and, by a hatbox argument, that $A$-almost everywhere on the surface $\mathscr{S}$

$$
-\mathbf{T}_{+} \mathbf{N}=\mathbf{t}_{\mathscr{B}+}+\mathbf{b}_{\mathscr{B}+}
$$

and

$$
\mathbf{T}_{-} \mathbf{N}=\mathbf{t}_{\mathscr{B}-}+\mathbf{b}_{\mathscr{B}-} \cdot
$$

The two equations on $\mathscr{S}$ combine to create the jump condition:

$$
\left(\mathbf{T}_{-}-\mathbf{T}_{+}\right) \mathbf{N}=-\left(\mathbf{t}_{\mathscr{B}-}+\mathbf{t}_{\mathscr{B}+}\right)+\left(\mathbf{b}_{\mathscr{B}-}+\mathbf{b}_{\mathscr{B}+}\right) .
$$

For parts of the surface we get $^{8}$

$$
\int_{\partial \mathscr{R}} \mathbf{t}_{\partial \mathscr{R}} d L+\int_{\mathscr{R}} \mathbf{t}_{\mathscr{S}_{+}}+\mathbf{t}_{\mathscr{S}_{-}}+\mathbf{b}_{\mathscr{S}} d A=\mathbf{0}
$$

and may apply arguments of Gurtin and Murdoch ([GMu], also cf. [P]) to show results similar to the above result: there exists a stress tensor field $\mathbf{T}_{\mathscr{S}}$, which is symmetric and tangential (defined on tangent vectors and tangent-vector valued), such that

$$
\operatorname{div}_{\mathscr{S}} \mathbf{T}_{\mathscr{S}}+\mathbf{t}_{\mathscr{S}_{+}}+\mathbf{t}_{\mathscr{S}_{-}}+\mathbf{b}_{\mathscr{S}}=\mathbf{0}, \quad \mathbf{T}_{\mathscr{S}}^{\mathrm{T}} \boldsymbol{\nu}=\mathbf{t}_{\partial \mathscr{R}}
$$

where $\nu$ is the exterior normal to $\mathscr{R}$ (in the tangent space) and $\operatorname{div}_{\mathscr{S}}$ is the surface divergence. However, as we have remarked (cf. [P]), the forces acting on $\mathbf{v}$ are all normal, so $\mathbf{T}_{\mathscr{S}}$ is both normal and tangential, and thus

$$
\mathbf{T}_{\mathscr{S}}=\mathbf{0} \text {. }
$$

Hence the surface force balance reduces to

$$
\mathbf{t}_{\mathscr{S}_{+}}+\mathbf{t}_{\mathscr{S}_{-}}+\mathbf{b}_{\mathscr{S}}=\mathbf{0} \text {. }
$$

The interaction-workings between $\mathscr{B}_{ \pm}$and $\mathscr{S}$ yield

$$
\begin{aligned}
\mathbf{t}_{\mathscr{B}+}+\mathbf{t}_{\mathscr{S}_{+}}=\mathbf{0}, \\
\mathbf{t}_{\mathscr{B}-}+\mathbf{t}_{\mathscr{S}_{-}}=\mathbf{0},
\end{aligned}
$$

\footnotetext{
${ }^{8}$ The fact that we require the velocity $\mathbf{v}$ to be normal to the surface does not affect Murdoch and Gurtin's computations, as is shown by Pfenning [Pf, pp. 66-68].
} 
which can be combined with the other balance equations to yield the equation

$$
\left[T_{+}-T_{-}\right] \mathbf{N}+\mathbf{b}_{\mathscr{S}}=-\mathbf{b}_{\mathscr{B}+}-\mathbf{b}_{\mathscr{B}-}
$$

or

$$
\left[T_{+}-T_{-}\right] \mathbf{N}+\mathbf{b}_{\mathscr{S}}=\mathbf{M}\left(\mathbf{v}_{-}-\mathbf{v}_{+}\right){ }^{9}
$$

This is the jump-equation for the stresses. Leaving aside the body force on the surface, the last equation expresses that the jump in the stresses across the surface balances the momentum transfer. This result arises because the surface stress is zero and is exactly that predicted in the classical formulation [W, p. 234]. Surface stress effects can enter through the other interaction terms, as we shall see in the next section.

7. Material balance of forces. The interaction terms involving relative velocities are novel. Their presence here formally is due to our modeling the surface-bulk aggregate as a structured continuum. Physically, they represent the power expended to change the shape of the surface and, thus, are central to the theory. Since these terms do not fall into the traditional form of continuum mechanics, there is no guide to the construction of the corresponding interaction forces. ${ }^{10}$ Gurtin and Struthers [GS] re-express the representation in the reference configuration of one of the bulk phases, e.g., $\mathscr{B}_{+}$, and assume that the working is invariant under superposition of rigid motions on $\mathbf{v}-\mathbf{v}_{+}$; they refer to this as lattice frame indifference. We prefer to take another point of view, regarding any such requirement of invariance under modification of the relative velocities as an expression of material symmetry. We consider a thought-experiment to illustrate our argument: suppose that the body $\mathscr{B}_{-}$ consists of a crystalline solid phase, say in a simple cubic lattice, while the body $\mathscr{B}_{+}$ is a liquid phase, and that the surface of separation moves parallel to itself into the liquid so that deposition is occurring on the solid. After a certain time consider the situation: the surface has exactly the same form, the only change in energy being the cost of attachment of a particle to the crystal. This energy expense may be regarded as an exchange of energy between the phases; in particular, neither $P\left(\mathscr{B}_{+}, \mathscr{S}, V\right)$ nor $P\left(\mathscr{B}_{-}, \mathscr{S}, V\right)$ need be zero, but the net exchanges $P\left(\mathscr{B}_{+}, \mathscr{S}, V\right)+P(\mathscr{S}, \mathscr{B}+V)$ and $P\left(\mathscr{B}_{-}, \mathscr{S}, V\right)+P\left(\mathscr{S}, \mathscr{B}_{-}, V\right)$ will be zero. Also, there is no power required to change the shape of the surface; it has not changed; hence, $P\left(\mathscr{S}, \mathscr{S}^{\mathrm{e}}, V\right)$ is zero. We might consider also a rigid rotation: if the surface is rotated thereby in its own plane, then there is no essential change in the surface, but if the rotation is out of this plane then the orientation of the surface relative to the lattice is changed, and we must say that the surface has been altered. In particular, it is clear that the structure of the lattice is very significant in this argument: if each phase were amorphous, we

\footnotetext{
${ }^{9}$ In the interaction balances involving elements $\mathscr{P}$ of the body and $\mathscr{R}$ of the surface, one might wonder at the absence of terms representing the transport of area of $\mathscr{R}$ through the boundary of $\mathscr{P}$ [FDW, GSW]. In formulating the balance it suffices to consider first $\mathscr{P}$ for which no such terms occur: the resulting detailed balance then ensures that the transport terms in the general case will be zero. The only exception is the case in which $\mathscr{P}=\mathscr{B}$, which generates new boundary terms.

${ }^{10}$ See Capriz's monograph [C] for a discussion of the form which such balances usually take in the theory of continua with microstructure. In fact, we believe that our methods as presented here should prove useful in these contexts.
} 
would expect invariance under the full group of rotations. (In addition, it is clear that these arguments are to be applied only locally; the relation of the rotation to the local tangent plane is what is significant.) It is clear, however, that invariance under the group of translations is always to be expected. We treat the consequences of that first, deferring the more involved discussion of invariance under rotations. To summarize: under transformations, which consist of a rigid translation of the surface relative to both bulk segments, we will suppose that each of

$$
P(\mathscr{P}, \mathscr{R}, V)+P(\mathscr{R}, \mathscr{P}, V), P\left(\mathscr{R}, \mathscr{R}^{\mathrm{e}}, V\right)
$$

is unchanged, for all parts $\mathscr{R}$ of $\mathscr{S}$ and $\mathscr{P}$ of $\mathscr{B}_{ \pm}$.

Suppose that the velocity of the surface is modified by any rigid translation c (equivalent, in light of the invariance under rigid motions, to allowing both $\mathbf{v}_{+}$and $\mathbf{v}_{-}$to be modified by $-\mathbf{c}$ )

$$
V=\left(\mathbf{v}, \mathbf{v}_{+}, \mathbf{v}_{-}\right) \mapsto\left(\mathbf{v}+\mathbf{c}, \mathbf{v}_{+}, \mathbf{v}_{-}\right),
$$

and consider the effect on the terms

$$
\begin{aligned}
P(\mathscr{P}, \mathscr{R}, V)+P(\mathscr{R}, \mathscr{P}, V)= & \int_{\mathscr{P} \cap \mathscr{R}} \mathbf{t}_{\mathscr{B}^{ \pm}} \cdot \mathbf{v}_{ \pm}+\boldsymbol{\tau}_{\mathscr{B}^{ \pm}} \cdot\left(\mathbf{v}-\mathbf{v}_{ \pm}\right) d A, \\
& +\int_{\mathscr{R} \cap \mathscr{P}} \mathbf{t}_{\mathscr{S}^{ \pm}} \cdot \mathbf{v}+\boldsymbol{\tau}_{\mathscr{S}^{ \pm}} \cdot\left(\mathbf{v}-\mathbf{v}_{ \pm}\right) d A
\end{aligned}
$$

and

$$
\begin{aligned}
P\left(\mathscr{R}, \mathscr{R}^{\mathrm{e}}, V\right)= & \int_{\partial \mathscr{R}} \mathbf{t}_{\partial \mathscr{R}} \cdot \mathbf{v}+\boldsymbol{\tau}_{\partial \mathscr{R}}^{+} \cdot\left(\mathbf{v}-\mathbf{v}_{+}\right)+\boldsymbol{\tau}_{\partial \mathscr{R}}^{-} \cdot\left(\mathbf{v}-\mathbf{v}_{-}\right) d L \\
& +\int_{\mathscr{R}} \mathbf{b}_{\mathscr{S}} \cdot \mathbf{v}+\boldsymbol{\beta}_{\mathscr{S}_{+}} \cdot\left(\mathbf{v}-\mathbf{v}_{+}\right)+\boldsymbol{\beta}_{\mathscr{S}_{-}} \cdot\left(\mathbf{v}-\mathbf{v}_{-}\right) \\
& +\mathbf{t}_{\mathscr{S}_{+}} \cdot \mathbf{v}+\boldsymbol{\tau}_{\mathscr{S}_{+}} \cdot\left(\mathbf{v}-\mathbf{v}_{+}\right)+\mathbf{t}_{\mathscr{S}_{-}} \cdot \mathbf{v}+\boldsymbol{\tau}_{\mathscr{S}_{-}} \cdot\left(\mathbf{v}-\mathbf{v}_{-}\right) d A .
\end{aligned}
$$

Of course some terms vanish because of the balance of forces; those that remain are

$$
\begin{gathered}
\mathbf{c} \cdot\left[\int_{\mathscr{P} \cap \mathscr{R}} \boldsymbol{\tau}_{\mathscr{B}^{ \pm}}+\mathbf{t}_{\mathscr{S}^{ \pm}}+\boldsymbol{\tau}_{\mathscr{S}^{ \pm}} d A\right]=0, \\
\mathbf{c} \cdot\left[\int_{\partial \mathscr{R}} \boldsymbol{\tau}_{\partial \mathscr{R}}^{+}+\boldsymbol{\tau}_{\partial \mathscr{R}}^{-} d L+\int_{\mathscr{R}} \boldsymbol{\tau}_{\mathscr{S}_{+}}+\boldsymbol{\tau}_{\mathscr{S}_{-}}+\boldsymbol{\beta}_{\mathscr{S}_{+}}+\boldsymbol{\beta}_{\mathscr{S}_{-}} d A\right]=0 .
\end{gathered}
$$

Since the first of these is required to hold for all $\mathbf{c}$ :

$$
\boldsymbol{\tau}_{\mathscr{B}^{ \pm}}+\mathbf{t}_{\mathscr{S}^{ \pm}}+\boldsymbol{\tau}_{\mathscr{S}^{ \pm}}=\mathbf{0} .
$$

Similarly, using the arguments already mentioned in the previous section, we discover that the second implies the existence of a material stress tensor field $\Upsilon$ defined on tangent vectors such that

$$
\operatorname{div}_{\mathscr{S}} \boldsymbol{\Upsilon}+\boldsymbol{\tau}_{\mathscr{S}_{+}}+\boldsymbol{\tau}_{\mathscr{S}_{-}}=\mathbf{0}, \quad \boldsymbol{\Upsilon}^{\mathrm{T}} \boldsymbol{\nu}=\boldsymbol{\tau}_{\partial \mathscr{R}}^{+}+\boldsymbol{\tau}_{\partial \mathscr{R}}^{-} .
$$

The material force balance equation reduces by use of the above relations and by use of the corresponding balance of forces to

$$
\operatorname{div}_{\mathscr{S}} \mathbf{\Upsilon}+\mathbf{b}_{\mathscr{S}}=\mathbf{0}
$$


or

$$
\operatorname{div}_{\mathscr{S}} \mathbf{\Upsilon}-\left[\mathbf{T}_{+}-\mathbf{T}_{-}\right] \mathbf{N}-\boldsymbol{\tau}_{\mathscr{B}+}-\boldsymbol{\tau}_{\mathscr{B}-}=\mathbf{M}\left(\mathbf{v}_{+}-\mathbf{v}_{-}\right) .
$$

This forms our fundamental equation of balance of surface forces.

Finally, let us remark that if we assume that the surface is isotropic, so that the local working is invariant under local tangent-plane rotations, it would follow that

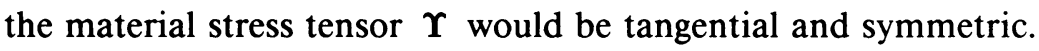

8. Balance of energy. For use in the following equations we need to examine the form that the net working takes in light of the various representations and assumptions of the previous sections. First,

$$
\begin{aligned}
P\left(\mathscr{P}, \mathscr{P}^{\mathrm{e}}, V\right)= & \int_{\partial \mathscr{P} \backslash \mathscr{S}} \mathbf{T}_{ \pm} \mathbf{n} \cdot \mathbf{v}_{ \pm} d A+\int_{\mathscr{P}} \mathbf{b} \cdot \mathbf{v}_{ \pm} d V \\
& +\int_{\mathscr{P} \cap \mathscr{S}} \mathbf{t}_{\mathscr{B}^{ \pm}} \cdot \mathbf{v}_{ \pm}+\boldsymbol{\tau}_{\mathscr{B}^{ \pm}} \cdot\left(\mathbf{v}-\mathbf{v}_{ \pm}\right)+\mathbf{b}_{\mathscr{B}^{ \pm}} \cdot \mathbf{v}_{ \pm}+\boldsymbol{\beta}_{\mathscr{B}}^{ \pm} \cdot\left(\mathbf{v}-\mathbf{v}_{ \pm}\right) d A \\
= & \int_{\mathscr{P}} \mathbf{T}_{ \pm} \cdot \nabla_{ \pm} \mathbf{v}_{ \pm} d V+\int_{\mathscr{P} \cap \mathscr{S}} \boldsymbol{\tau}_{\mathscr{B}^{ \pm}} \cdot\left(\mathbf{v}-\mathbf{v}_{ \pm}\right) d A
\end{aligned}
$$

Then

$$
\begin{aligned}
P(\mathscr{P}, \mathscr{R}, V)+P(\mathscr{R}, \mathscr{P}, V)= & \int_{\mathscr{P} \cap \mathscr{R}} \mathbf{t}_{\mathscr{B}^{ \pm}} \cdot \mathbf{v}_{ \pm}+\boldsymbol{\tau}_{\mathscr{B}^{ \pm}} \cdot\left(\mathbf{v}-\mathbf{v}_{ \pm}\right) d A \\
& +\int_{\mathscr{R} \cap \mathscr{P}} \mathbf{t}_{\mathscr{S}^{ \pm}} \cdot \mathbf{v}+\boldsymbol{\tau}_{\mathscr{S}^{ \pm}} \cdot\left(\mathbf{v}-\mathbf{v}_{ \pm}\right) d A \\
= & \int_{\mathscr{R} \cap \mathscr{P}} \mathbf{t}_{\mathscr{B}^{ \pm}} \cdot \mathbf{v}_{ \pm}+\mathbf{t}_{\mathscr{S}^{ \pm}} \cdot \mathbf{v}_{ \pm} d A \\
= & 0 .
\end{aligned}
$$

This means that under our assumptions, there is no net exchange of power between the bulk and the surface elements.

Finally,

$$
\begin{aligned}
P\left(\mathscr{R}, \mathscr{R}^{\mathrm{e}}, V\right)= & \int_{\partial \mathscr{R}} \mathbf{t}_{\partial \mathscr{R}} \cdot \mathbf{v}+\boldsymbol{\tau}_{\partial \mathscr{R}}^{+} \cdot\left(\mathbf{v}-\mathbf{v}_{+}\right)+\boldsymbol{\tau}_{\partial \mathscr{R}}^{-} \cdot\left(\mathbf{v}-\mathbf{v}_{-}\right) d L \\
& +\int_{\mathscr{R}} \mathbf{b}_{\mathscr{S}} \cdot \mathbf{v}+\boldsymbol{\beta}_{\mathscr{S}_{+}} \cdot\left(\mathbf{v}-\mathbf{v}_{+}\right)+\boldsymbol{\beta}_{\mathscr{S}-} \cdot\left(\mathbf{v}-\mathbf{v}_{-}\right) \\
& +\mathbf{t}_{\mathscr{S}_{+}} \cdot \mathbf{v}+\boldsymbol{\tau}_{\mathscr{S}+} \cdot\left(\mathbf{v}-\mathbf{v}_{+}\right)+\mathbf{t}_{\mathscr{P}-} \cdot \mathbf{v}+\boldsymbol{\tau}_{\mathscr{P}-} \cdot\left(\mathbf{v}-\mathbf{v}_{-}\right) d A \\
= & \int_{\mathscr{R}} \boldsymbol{\Upsilon}^{\mathrm{T}} \cdot \nabla_{\mathscr{S}}\left(\mathbf{v}-\mathbf{v}_{-}\right)+\boldsymbol{\tau}_{\mathscr{P}_{+}} \cdot\left(\mathbf{v}_{-}-\mathbf{v}_{+}\right) d A+\int_{\partial \mathscr{R}} \boldsymbol{\tau}_{\partial \mathscr{R}}^{+} \cdot\left(\mathbf{v}_{-}-\mathbf{v}_{+}\right) d L .
\end{aligned}
$$

Now we turn to the expression of the equation of balance of energy. We have supposed that $E$ (and hence $\dot{E}$ ) is additive and has bounds which ensure that the representations

$$
\begin{aligned}
& \dot{E}(\mathscr{P})=\int_{\mathscr{P}} \rho \dot{e} d V \mp \int_{\mathscr{P} \cap \mathcal{P}} M e_{ \pm} d A, \\
& \dot{E}(\mathscr{R})=\int_{\mathscr{R}} \stackrel{\circ}{\varepsilon}-\varepsilon \kappa \mathbf{v} \cdot \mathbf{N} d A
\end{aligned}
$$

are valid. $^{11}$

\footnotetext{
${ }^{11}$ In fact, it is possible to show that the additivity of $\dot{E}$ follows from the requirement of balance of energy, excepting only the cases $\mathscr{P} \cup \mathscr{K}$ and $\mathscr{P} \cup \mathscr{Q}$ in which the two parts in question span the surface. Thus our assumption of additivity can be regarded as being essential only for those cases (cf. [P, p. 23]).
} 
The governing balance equations now are

$$
\begin{gathered}
\dot{E}(\mathscr{P})=P\left(\mathscr{P}, \mathscr{P}^{\mathrm{e}}, V\right)+H\left(\mathscr{P}, \mathscr{P}^{\mathrm{e}}\right), \\
\dot{E}(\mathscr{R})=P\left(\mathscr{R}, \mathscr{R}^{\mathrm{e}}, V\right)+H\left(\mathscr{R}, \mathscr{R}{ }^{\mathrm{e}}\right), \\
0=P(\mathscr{P}, \mathscr{R}, V)+P(\mathscr{R}, \mathscr{P}, V)+H(\mathscr{P}, \mathscr{R})+H(\mathscr{R}, \mathscr{P}) .
\end{gathered}
$$

Balance of all combined parts is ensured by these equations, the assumptions of additivity, and the measure-bounds that we have assumed.

We now expand these equations, using all of the above representations. For the first, balance of energy for $\mathscr{P}$,

$$
\begin{aligned}
\int_{\mathscr{P}} \rho \dot{e} d V \mp \int_{\mathscr{P} \cap \mathscr{S}} M e_{ \pm} d A= & \int_{\mathscr{D}} \mathbf{T}_{ \pm} \cdot \nabla \mathbf{v}_{ \pm} d V+\int_{\mathscr{P} \cap \mathscr{S}^{\prime}} \boldsymbol{\tau}_{\mathscr{B}^{ \pm}} \cdot\left(\mathbf{v}-\mathbf{v}_{ \pm}\right) d A \\
& +\int_{\mathscr{P}} r d V+\int_{\partial \mathscr{P} \backslash \mathscr{S}} q_{\partial \mathscr{P}} d A+\int_{\mathscr{P} \cap \mathscr{S}} q_{\mathscr{B}^{ \pm}} d A .
\end{aligned}
$$

We can apply this to all $\mathscr{P}$ that do not intersect $\mathscr{S}$ and use standard arguments to deduce the existence of vector fields $\mathbf{q}_{ \pm}$on $\mathscr{B}_{ \pm}$that satisfy

$$
\mathbf{q}_{ \pm} \cdot \mathbf{n}=q_{\partial \mathscr{P}} \quad \text { on } \partial \mathscr{P} \backslash \mathscr{S} .
$$

Then we can see, as for the force representations, that $\mathbf{q}_{ \pm}$can be defined on $\mathscr{S}$ as well, where they satisfy

$$
\begin{aligned}
-\mathbf{q}_{+} \cdot \mathbf{N} & =q_{\mathscr{B}+}+M e_{+}+\tau_{\mathscr{B}+} \cdot\left(\mathbf{v}-\mathbf{v}_{+}\right), \\
\mathbf{q}_{-} \cdot \mathbf{N} & =q_{\mathscr{B}-}-M e_{-}+\boldsymbol{\tau}_{\mathscr{B}-} \cdot\left(\mathbf{v}-\mathbf{v}_{-}\right),
\end{aligned}
$$

and, as a consequence, the jump condition

$$
\left(\mathbf{q}_{-}-\mathbf{q}_{+}\right) \cdot \mathbf{N}=q_{\mathscr{B}+}+q_{\mathscr{B}-}+M\left(e_{+}-e_{-}\right)+\boldsymbol{\tau}_{\mathscr{B}+} \cdot\left(\mathbf{v}-\mathbf{v}_{+}\right)+\boldsymbol{\tau}_{\mathscr{B}-} \cdot\left(\mathbf{v}-\mathbf{v}_{-}\right) .
$$

This equation is of particular significance in the case of phase transitions; it relates the heat loss across the surface to the heat accepted by the surface (changed into surface energy, perhaps, or conducted away along the surface) and the latent heat of the phase change, $e_{+}-e_{-}$.

The usual smoothness assumptions applied to q lead to the equation

$$
\rho \dot{e}=\operatorname{div} \mathbf{q}_{ \pm}+\mathbf{T}_{ \pm} \cdot \nabla \mathbf{v}_{ \pm}+r,
$$

which represents the local balance of energy in bulk.

Next we consider the balance for the surface element $\mathscr{R}$,

$$
\begin{aligned}
\int_{\mathscr{R}} \stackrel{\circ}{\varepsilon}-\varepsilon \kappa \mathbf{v} \cdot \mathbf{N} d A= & \int_{\mathscr{R}} \Upsilon^{\mathrm{T}} \cdot \nabla_{\mathscr{S}}\left(\mathbf{v}-\mathbf{v}_{-}\right)+\boldsymbol{\tau}_{\mathscr{S}_{+}} \cdot\left(\mathbf{v}_{-}-\mathbf{v}_{+}\right)+q_{\mathscr{S}_{+}}+q_{\mathscr{S}_{-}}+r_{\mathscr{S}} d A \\
& +\int_{\partial \mathscr{R}} \boldsymbol{\tau}_{\partial \mathscr{R}}^{+} \cdot\left(\mathbf{v}_{-}-\mathbf{v}_{+}\right)+q_{\partial \mathscr{R}} d L
\end{aligned}
$$


Requiring that this must hold for all choices of $\mathscr{R}$ and noting that the area integrals are additive in $\mathscr{R}$, we may apply the usual sort of limiting argument (cf. [GMu, P]) first to reduce the form of the boundary contribution

$$
\boldsymbol{\tau}_{\partial \mathscr{R}}^{+} \cdot\left(\mathbf{v}_{-}-\mathbf{v}_{+}\right)+q_{\partial \mathscr{R}}
$$

to one that depends solely on the (in-surface) normal $\nu$ to $\partial \mathscr{R}$ and then to prove that it is linear as a function of that normal. We choose to make a simplifying assumption, that this reduction is separately valid for the two parts of the term. We then obtain a heat flux vector $\gamma$ and a surface tensor $\Gamma$, with range the tangent space but defined for all vectors, such that

$$
q_{\partial \mathscr{R}}=\gamma \cdot \nu, \quad \tau_{\partial \mathscr{H}}^{+}=\Gamma^{\mathrm{T}} \nu .
$$

(We choose the transpose in the latter to make the final form more convenient.) Then we have

$$
\begin{aligned}
\int_{\mathscr{P}} \stackrel{\circ}{\varepsilon}-\varepsilon \kappa \mathbf{v} \cdot \mathbf{N} d A= & \int_{\mathscr{R}} \boldsymbol{\Upsilon}^{\mathrm{T}} \cdot \nabla_{\mathscr{S}}\left(\mathbf{v}-\mathbf{v}_{-}\right)+\boldsymbol{\tau}_{\mathscr{P}_{+}} \cdot\left(\mathbf{v}_{-}-\mathbf{v}_{+}\right)+q_{\mathscr{P}_{+}}+q_{\mathscr{P}_{-}}+r_{\mathscr{P}} d A \\
& +\int_{\partial \mathscr{R}} \Gamma\left(\mathbf{v}_{-}-\mathbf{v}_{+}\right) \cdot \boldsymbol{\nu}+\gamma \cdot \boldsymbol{\nu} d L,
\end{aligned}
$$

and, assuming the usual smoothness, it follows that $\stackrel{\circ}{\varepsilon}-\varepsilon \kappa \mathbf{v} \cdot \mathbf{N}=\boldsymbol{\Upsilon}^{\mathbf{T}} \cdot \nabla_{\mathscr{S}}\left(\mathbf{v}-\mathbf{v}_{-}\right)+\boldsymbol{\tau}_{\mathscr{S}_{+}} \cdot\left(\mathbf{v}_{-}-\mathbf{v}_{+}\right)+\operatorname{div}_{\mathscr{P}}\left\{\boldsymbol{\Gamma}\left(\mathbf{v}_{-}-\mathbf{v}_{+}\right)\right\}+q_{\mathscr{S}_{+}}+q_{\mathscr{P}_{-}}+r_{\mathscr{P}}+\operatorname{div}_{\mathscr{P}} \gamma$. This is our fundamental equation of balance of energy on the surface; it is interesting to note that a working term enters which involves a "stress" that does not appear in either the force balance or the material force balance.

Finally, we consider the equation of balance between $\mathscr{P}$ and $\mathscr{R}$, obtaining

$$
\int_{\mathscr{P} \cap \mathscr{R}} q_{\mathscr{B}^{ \pm}}+q_{\mathscr{S}^{ \pm}} d A=0
$$

so that

$$
q_{\mathscr{B}^{ \pm}}+q_{\mathscr{S}^{ \pm}}=0 \text {. }
$$

This can be combined with the jump condition for the heat flux and the balance of energy for $\mathscr{R}$ to yield our first version of the energy balance for the surface:

$$
\begin{aligned}
\stackrel{\circ}{\varepsilon}-\varepsilon \kappa \mathbf{v} \cdot \mathbf{N}= & \boldsymbol{\Upsilon}^{\mathrm{T}} \cdot \nabla_{\mathscr{S}}\left(\mathbf{v}-\mathbf{v}_{-}\right)+\operatorname{div}_{\mathscr{P}}\left\{\boldsymbol{\Gamma}\left(\mathbf{v}_{-}-\mathbf{v}_{+}\right)\right\} \\
& +\boldsymbol{\tau}_{\mathscr{S}+} \cdot\left(\mathbf{v}_{-}-\mathbf{v}_{+}\right)+\boldsymbol{\tau}_{\mathscr{B}+} \cdot\left(\mathbf{v}-\mathbf{v}_{+}\right)+\boldsymbol{\tau}_{\mathscr{B}-} \cdot\left(\mathbf{v}-\mathbf{v}_{-}\right) \\
& +\left(\mathbf{q}_{+}-\mathbf{q}_{-}\right) \cdot \mathbf{N}+M\left(e_{+}-e_{-}\right)+r_{\mathscr{P}}+\operatorname{div}_{\mathscr{S}} \gamma .
\end{aligned}
$$

We can also put this in a more symmetric, if less compact, form. We use the equations of material balance of force and define

$$
\Gamma_{+}=-\Gamma, \quad \Gamma_{-}=\Gamma-\Upsilon
$$

in order to rewrite the work terms as follows:

$$
\begin{aligned}
\boldsymbol{\Upsilon}^{\mathrm{T}} \cdot \nabla_{\mathscr{S}}\left(\mathbf{v}-\mathbf{v}_{-}\right)+\operatorname{div}_{\mathscr{S}}\left\{\boldsymbol{\Gamma}\left(\mathbf{v}_{-}-\mathbf{v}_{+}\right)\right\}+\boldsymbol{\tau}_{\mathscr{S}+} \cdot\left(\mathbf{v}_{-}-\mathbf{v}_{+}\right) \\
\quad+\boldsymbol{\tau}_{\mathscr{B}+} \cdot\left(\mathbf{v}-\mathbf{v}_{+}\right)+\boldsymbol{\tau}_{\mathscr{B}-} \cdot\left(\mathbf{v}-\mathbf{v}_{-}\right) \\
=\boldsymbol{\Upsilon}^{\mathrm{T}} \cdot \nabla_{\mathscr{S}} \mathbf{v}+\operatorname{div}_{\mathscr{S}}\left\{\boldsymbol{\Gamma}_{+} \mathbf{v}_{+}+\boldsymbol{\Gamma}_{-} \mathbf{v}_{-}\right\}-\boldsymbol{\tau}_{\mathscr{S}_{+}} \cdot \mathbf{v}_{+}-\boldsymbol{\tau}_{\mathscr{S}} \cdot \mathbf{v}_{-} \\
\quad+\boldsymbol{\tau}_{\mathscr{B}+} \cdot\left(\mathbf{v}-\mathbf{v}_{+}\right)+\boldsymbol{\tau}_{\mathscr{B}-} \cdot\left(\mathbf{v}-\mathbf{v}_{-}\right)
\end{aligned}
$$


but from previous calculations,

$$
\boldsymbol{\tau}_{\mathscr{S}^{ \pm}}+\boldsymbol{\tau}_{\mathscr{B}^{ \pm}}-\mathbf{t}_{\mathscr{B}^{ \pm}}=\mathbf{0}
$$

and from the representation for $\mathbf{T}_{ \pm}$, we see that

$$
-\mathbf{t}_{\mathscr{B}+} \cdot \mathbf{v}_{+}-\mathbf{t}_{\mathscr{B}-} \cdot \mathbf{v}_{-}=\mathbf{b}_{\mathscr{B}+} \cdot \mathbf{v}_{+}+\mathbf{b}_{\mathscr{B}-} \cdot \mathbf{v}_{-}+T_{+} \mathbf{N} \cdot \mathbf{v}_{+}+T_{-} \mathbf{N} \cdot \mathbf{v}_{-} \cdot
$$

The identification

$$
\mathbf{b}_{\mathscr{B}^{ \pm}}=\frac{1}{2} M \mathbf{v}_{ \pm}, \quad M=\rho_{-}\left(\mathbf{v}-\mathbf{v}_{-}\right) \cdot \mathbf{N},
$$

of the momentum transfer terms will complete the expression. We then insert that form into the energy equation to find the final form of the energy equation

$$
\begin{aligned}
\stackrel{\circ}{\varepsilon}-\varepsilon \kappa \mathbf{v} \cdot \mathbf{N}= & \boldsymbol{\Upsilon}^{\mathrm{T}} \cdot \nabla_{\mathscr{S}} \mathbf{v}+\operatorname{div}_{\mathscr{S}}\left\{\boldsymbol{\Gamma}_{+} \mathbf{v}_{+}+\boldsymbol{\Gamma}_{-} \mathbf{v}_{-}\right\}+\left(\boldsymbol{\tau}_{\mathscr{B}+}+\boldsymbol{\tau}_{\mathscr{B}-}\right) \cdot \mathbf{v} \\
& +r_{\mathscr{S}}+\operatorname{div}_{\mathscr{S}} \gamma+T_{+} \mathbf{N} \cdot \mathbf{v}_{+}-T_{-} \mathbf{N} \cdot \mathbf{v}_{-}+\left(\mathbf{q}_{+}-\mathbf{q}_{-}\right) \cdot \mathbf{N} \\
& +\rho_{-}\left[e_{+}-e_{-}+\frac{1}{2} \mathbf{v}_{+}^{2}-\frac{1}{2} \mathbf{v}^{2}\right]\left(\mathbf{v}-\mathbf{v}_{-}\right) \cdot \mathbf{N},
\end{aligned}
$$

with

$$
\mathbf{\Upsilon}+\boldsymbol{\Gamma}_{+}+\boldsymbol{\Gamma}_{-}=\mathbf{0} .
$$

This equation is more symmetric than the previous one and brings into focus the nature of the terms. The terms on the right-hand side express the work done to reshape the surface:

$$
\boldsymbol{\Upsilon}^{\mathrm{T}} \cdot \nabla_{\mathscr{S}} \mathbf{v}+\operatorname{div}_{\mathscr{S}}\left\{\boldsymbol{\Gamma} \cdot \mathbf{v}_{+}+\boldsymbol{\Gamma}_{-} \cdot \mathbf{v}_{-}\right\}
$$

the work done to translate the surface:

$$
\left(\boldsymbol{\tau}_{\mathscr{B}+}+\boldsymbol{\tau}_{\mathscr{B}-}\right) \cdot \mathbf{v}
$$

and the heat flux along the surface and the external heat supply into the surface:

$$
r_{\mathscr{S}}+\operatorname{div}_{\mathscr{S}} \gamma
$$

while the terms involving the bulk quantities and the surface effects, namely,

$$
T_{+} \mathbf{N} \cdot \mathbf{v}_{+}-T_{-} \mathbf{N} \cdot \mathbf{v}_{-}+\left(\mathbf{q}_{+}-\mathbf{q}_{-}\right) \cdot \mathbf{N}+\rho_{-}\left[e_{+}-e_{-}+\frac{1}{2} \mathbf{v}_{+}^{2}-\frac{1}{2} \mathbf{v}^{2}\right]\left(\mathbf{v}-\mathbf{v}_{-}\right) \cdot \mathbf{N},
$$

represent the apparent loss of energy across the surface.

9. Entropy inequality. We shall not approach the entropy inequality at the same fundamental level as the other equations: we shall introduce a (single) positive-valued temperature field $\theta$, presumed defined over the entire body, including $\mathscr{S}$, and express the entropy flux $F$ in terms of the heat flux and this field by

$$
\begin{aligned}
F(\mathscr{P}, \mathscr{Q}) & =\int_{\partial \mathscr{P} \cap \mathscr{Q}} \frac{\mathbf{q} \cdot \mathbf{n}}{\theta} d A, \\
F\left(\mathscr{P}, \mathscr{B}^{\mathrm{e}}\right) & =\int_{\partial \mathscr{P} \cap \mathscr{B}^{\mathrm{e}}} \frac{\mathbf{q} \cdot \mathbf{n}}{\theta} d A+\int \frac{r}{\theta} d V, \\
F(\mathscr{P}, \mathscr{R}) & =\int_{\mathscr{P} \cap \mathscr{R}} \frac{q_{\mathscr{R}}^{ \pm}}{\theta} d A, \quad F(\mathscr{R}, \mathscr{P})=\int_{\mathscr{R} \cap \mathscr{P}} \frac{q_{\mathscr{S}^{ \pm}}}{\theta} d A, \\
F(\mathscr{R}, \mathscr{T}) & =\int_{\mathscr{R} \cap \mathscr{T}} \frac{\gamma \cdot \mathbf{n}}{\theta} d L, \\
F\left(\mathscr{R}, \mathscr{B}^{\mathrm{e}}\right) & =\int_{\mathscr{R} \cap \mathscr{B}^{\mathrm{e}}} \frac{\gamma \cdot \mathbf{n}}{\theta} d L+\int_{\mathscr{R}} \frac{r_{\mathscr{S}}}{\theta} d A .
\end{aligned}
$$


Then the complete expression of the entropy inequality is

$$
\dot{S}(\mathscr{P}) \geq F\left(\mathscr{P}, \mathscr{P}^{\mathrm{e}}\right), \quad \dot{S}(\mathscr{R}) \geq F\left(\mathscr{R}, \mathscr{R}^{\mathrm{e}}\right),
$$

since the other appropriate inequalities, such as $0 \leq F(\mathscr{P}, \mathscr{R})+F(\mathscr{R}, \mathscr{P})$, are guaranteed, as equalities, by the corresponding results that we obtained for the heat flux.

Consider the first of the inequalities:

$$
\int_{\mathscr{P}} \rho \dot{s} d V \mp \int_{\mathscr{P} \cap \mathscr{S}} M s_{ \pm} d A \geq \int_{\partial \mathscr{P} \backslash \mathscr{S}} \frac{\mathbf{q} \cdot \mathbf{n}}{\theta} d A+\int_{\mathscr{P} \cap \mathscr{S}} \frac{q_{\mathscr{B}} \pm}{\theta} d A+\int_{\mathscr{P}} \frac{r}{\theta} d V .
$$

This inequality results in the local versions

$$
\rho \dot{s} \geq \operatorname{div}\left(\frac{\mathbf{q}}{\theta}\right)+\frac{r}{\theta}
$$

in the bulk, and

$$
\mp M s_{ \pm} \geq \mp \frac{\mathbf{q}_{ \pm} \cdot \mathbf{N}}{\theta}+\frac{q_{\mathscr{B}}}{\theta}
$$

on the surface. Combining the + and - versions of the latter inequality, we obtain the jump relation

$$
M\left(s_{-}-s_{+}\right) \geq \frac{\left(\mathbf{q}_{+}-\mathbf{q}_{-}\right) \cdot \mathbf{N}+\left(q_{\mathscr{B}+}+q_{\mathscr{B}-}\right)}{\theta} .
$$

We will find it useful to rewrite this using the jump relation for the energy:

$$
M\left(s_{-}-s_{+}\right) \geq \frac{M\left(e_{-}-e_{+}\right)-\tau_{\mathscr{B}+} \cdot\left(\mathbf{v}-\mathbf{v}_{+}\right)-\tau_{\mathscr{B}-} \cdot\left(\mathbf{v}-\mathbf{v}_{-}\right)}{\theta} .
$$

Looking at the inequality for the surface, we find

$$
\int_{\mathscr{R}} \stackrel{\circ}{\eta}-\eta \kappa \mathbf{v} \cdot \mathbf{n} d A \geq \int_{\partial \mathscr{R}} \frac{\gamma \cdot \mathbf{n}}{\theta} d L+\int_{\mathscr{R}} \frac{q_{\mathscr{S}_{+}}+q_{\mathscr{S}_{-}}}{\theta}+\frac{r_{\mathscr{S}}}{\theta} d A,
$$

which can be reduced to the local form

or

$$
\stackrel{\circ}{\eta}-\eta \kappa \mathbf{v} \cdot \mathbf{N} \geq \operatorname{div}_{\mathscr{S}}\left(\frac{\gamma}{\theta}\right)+\frac{q_{\mathscr{S}_{+}}+q_{\mathscr{S}_{-}}+r_{\mathscr{S}}}{\theta}
$$

Since

$$
\stackrel{\circ}{\eta}-\eta \kappa \mathbf{v} \cdot \mathbf{N} \geq \frac{\operatorname{div}_{\mathscr{S}} \gamma+r_{\mathscr{S}}}{\theta}+\frac{q_{\mathscr{S}+}+q_{\mathscr{S}_{-}}}{\theta}-\frac{\gamma \cdot \nabla_{\mathscr{S}} \theta}{\theta^{2}} \text {. }
$$

we may combine this with the jump equation to give

$$
\stackrel{\circ}{\eta}-\eta \kappa \mathbf{v} \cdot \mathbf{N}+M\left(s_{-}-s_{+}\right) \geq \frac{\operatorname{div}_{\mathscr{S}} \gamma+r_{\mathscr{S}}}{\theta}+\frac{\left(\mathbf{q}_{+}-\mathbf{q}_{-}\right) \cdot \mathbf{N}}{\theta}-\frac{\gamma \cdot \nabla_{\mathscr{S}} \boldsymbol{\theta}}{\theta^{2}},
$$

which is a combined entropy inequality for the surface.

The entropy equations can be combined with the energy balances to devise Claussius-Duhem relations, which serve to determine constitutive relations. The bulk equations follow the standard reduction and become

$$
\rho \dot{\psi}+\rho s \dot{\theta} \leq \mathbf{T} \cdot \nabla \mathbf{v}_{ \pm}+\frac{1}{\theta} \mathbf{q} \cdot \nabla \theta,
$$

where $\psi:=e-\theta s$ is the free energy. 
The jump equation can be re-expressed by use of the jump equation for the energy

$$
\theta M\left(s_{-}-s_{+}\right) \geq M\left(e_{-}-e_{+}\right)-\tau_{\mathscr{B}+} \cdot\left(\mathbf{v}-\mathbf{v}_{+}\right)-\tau_{\mathscr{B}-} \cdot\left(\mathbf{v}-\mathbf{v}_{-}\right)
$$

or, finally,

$$
M\left(\psi_{-}-\psi_{+}\right) \leq \boldsymbol{\tau}_{\mathscr{B}+} \cdot\left(\mathbf{v}-\mathbf{v}_{+}\right)+\boldsymbol{\tau}_{\mathscr{B}-} \cdot\left(\mathbf{v}-\mathbf{v}_{-}\right) .
$$

For the surface equations, we can eliminate $q_{\mathscr{S}_{+}}+q_{\mathscr{S}_{-}}+\operatorname{div}_{\mathscr{S}} \gamma+r_{\mathscr{S}}$ by using the energy equation to obtain

$$
\begin{aligned}
\stackrel{\circ}{\eta}-\eta \kappa \mathbf{v} \cdot \mathbf{N} \geq & \stackrel{\circ}{\varepsilon}-\varepsilon \kappa \mathbf{v} \cdot \mathbf{N}-\boldsymbol{\Upsilon}^{\mathrm{T}} \cdot \nabla_{\mathscr{S}}\left(\mathbf{v}-\mathbf{v}_{-}\right)-\boldsymbol{\tau}_{\mathscr{S}_{+}} \cdot\left(\mathbf{v}_{-}-\mathbf{v}_{+}\right) \\
& -\operatorname{div}_{\mathscr{S}}\left\{\boldsymbol{\Gamma}\left(\mathbf{v}_{-}-\mathbf{v}_{+}\right)\right\}-\frac{1}{\theta} \gamma \cdot \nabla_{\mathscr{S}} \theta,
\end{aligned}
$$

which then becomes

$$
\begin{aligned}
\stackrel{\circ}{\omega}+\eta \stackrel{\circ}{\theta}-\omega \kappa \mathbf{v} \cdot \mathbf{N} \leq & \boldsymbol{\Upsilon}^{\mathrm{T}} \cdot \nabla_{\mathscr{S}}\left(\mathbf{v}-\mathbf{v}_{-}\right)+\boldsymbol{\tau}_{\mathscr{S}_{+}} \cdot\left(\mathbf{v}_{-}-\mathbf{v}_{+}\right) \\
& +\operatorname{div}_{\mathscr{S}}\left\{\boldsymbol{\Gamma}\left(\mathbf{v}_{-}-\mathbf{v}_{+}\right)\right\}+\frac{1}{\theta} \boldsymbol{\gamma} \cdot \nabla_{\mathscr{S}} \boldsymbol{\theta}
\end{aligned}
$$

if we set $\omega:=\varepsilon-\theta \eta$ to be the surface free energy. An alternative form is generated by the second version of the energy balance for the surface

$$
\begin{aligned}
\stackrel{\circ}{\omega}+\eta \stackrel{\circ}{\theta}-\omega \kappa \mathbf{v} \cdot \mathbf{N} \leq & \boldsymbol{\Upsilon}^{\mathrm{T}} \cdot \nabla_{\mathscr{S}} \mathbf{v}+\operatorname{div}_{\mathscr{S}}\left\{\boldsymbol{\Gamma}_{+} \mathbf{v}_{+}+\boldsymbol{\Gamma}_{-} \mathbf{v}_{-}\right\} \\
& +\boldsymbol{\tau}_{\mathscr{B}+} \cdot \mathbf{v}_{+}+\boldsymbol{\tau}_{\mathscr{B}-} \cdot \mathbf{v}_{-}+T_{+} \mathbf{N} \cdot \mathbf{v}_{+}-T_{-} \mathbf{N} \cdot \mathbf{v}_{-} \\
& +\rho_{-}\left[\frac{1}{2} \mathbf{v}_{+}^{2}-\frac{1}{2} \mathbf{v}^{2}\right]\left(\mathbf{v}-\mathbf{v}_{-}\right) \cdot \mathbf{N}+\frac{1}{\theta} \gamma \cdot \nabla_{\mathscr{S}} \boldsymbol{\theta} .
\end{aligned}
$$

We recall as before that $\boldsymbol{\Upsilon}+\boldsymbol{\Gamma}_{+}+\boldsymbol{\Gamma}_{-}=\mathbf{0}$.

Acknowledgments. The research described in this paper was partially supported by the National Science Foundation. We are grateful to M. Gurtin and A. Struthers for comments on an earlier draft of the paper.

\section{REFERENCES}

[C] G. Capriz, Continua with Microstructure, Springer Tracts in Natural Philosophy 35, SpringerVerlag, New York, 1989

[FDW] J. Fernandez-Diaz and W. O. Williams, A generalized Stefan condition, Z. Angew. Math. Phys. 30, 749-755 (1979)

[FL] G. M. C. Fischer and M. H. Leitman, On continuum thermodynamics with surfaces, Arch. Rational Mech. Anal. 30, 225-262 (1968)

[G] M. E. Gurtin, On thermomechanical laws for the motion of a phase interface, Z. Angew. Math. Phys. 42, 370-388 (1991)

[GMa] M. E. Gurtin and L.-C. Martins, Cauchy's theorem in classical physics, Arch. Rational Mech. Anal. 60, 305-324 (1976)

[GMu] M. E. Gurtin and A. I. Murdoch, A continuum theory of elastic material surfaces, Arch. Rational Mech. Anal. 50, 291-323 (1974)

[GR] A. E. Green and R. S. Rivlin, Simple force and stress multipoles, Arch. Rational Mech. Anal. 16, 325-353 (1964)

[GS] M. E. Gurtin and A. Struthers, Phase boundaries in the presence of bulk deformation, Arch. Rational Mech. Anal. 112, 97-160 (1990)

[GSW] M. E. Gurtin, A. Struthers, and W. O. Williams, A transport theorem for moving interfaces, Quart. Appl. Math. 47, 773-777 (1989) 
[GW] M. E. Gurtin and W. O. Williams, An axiomatic foundation for continuum thermodynamics, Arch. Rational Mech. Anal. 26, 83-117 (1967)

[GWZ] M. E. Gurtin, W. O. Williams, and W. P. Ziemer, Geometric measure theory and the axioms for continuum thermodynamics, Arch. Rational Mech. Anal. 92, 1-22 (1986)

[M] G. P. Moeckel, Thermodynamics of an interface, Arch. Rational Mech. Anal. 57, 255-280 (1975)

[N1] W. Noll, The foundation of classical mechanics in the light of recent advances in continuum mechanics, The Axiomatic Method, with Special Reference to Geometry and Physics, North-Holland, Amsterdam, 1959, pp. 266-281

[N2] W. Noll, La mechanique classique, basée sur un axiome d'objetivitie, La Methode Axiomatique dans les Mecaniques Classiques et Nouvelles, Gauthier-Villars, Paris, 1963, pp. 47-56

[P] N. M. Pfenning, Theory of non-material surfaces with energy concentration, Ph.D. Thesis, Carnegie Mellon Univ., 1984

[S1] M. Šilhavý, The existence of the flux vector and the divergence theorem for general Cauchy fluxes, Arch. Rational Mech. Anal. 90, 195-212 (1985)

[S2] M. Šilhavý, Cauchy's stress theorem and tensor fields with divergences in $L^{p}$, Arch. Rational Mech. Anal. 116, 223-255 (1991)

[W] W. O. Williams, On work and energy in general continuua, II. Surfaces of discontinuity, Arch. Rational Mech. Anal. 49, 225-240 (1972) 